\title{
THE BASIC THEOREMS OF NATURAL SELECTION : A NAÏVE APPROACH
}

JOHN R. G. TURNER

Department of Biology, University of York

Received 8.iii.68

\section{INTRODUGTION}

REGENTLY both Fisher's "fundamental theorem of natural selection " (that the mean fitness of a population tends always to increase, subject to the restraints of overpopulation and change of environment) (Fisher, 1930) and Wright's "adaptive topography" (a graph of mean fitness in which the tendency for mean fitness to maximise is used to predict equilibria and so on) (see for example Lewontin and White, 1958; Li, 1955a) have been much criticised. The fundamental theorem is true only with random mating and with constant selection which is the same in both sexes, and is even then only an approximation if there is dominance ( $\mathrm{Li}, 1967 a, c$; Turner, 1967b). It is in general untrue if there is more than one locus (Moran, 1964; Turner, 1967b). The adaptive topography is more robust, as a more complex version may be used for pairs of loci with epistasis (Turner, 1967a) and one can devise parameters, more elaborate than mean fitness, which will maximise under many systems of selection and mating (see for example, Wright, 1955; Li, 1955a, 1967b). However, their complexity and lack of physical meaning makes these parameters inelegant; one feels that there must be a basic theorem embracing them all. This paper attempts such a theorem. The parameter used is comparatively simple, and the theorem must be known already in some form to almost every population geneticist; but so far it has been little used, except in relation to heritability in quantitative genetics (Falconer, 1961).

First the theorem will be expounded for selection, the same in both sexes, on single autosomal loci, in diploids. Then some special cases will be derived, and finally the theorem will be extended to some less restricted cases.

\section{THE THEOREM}

If $q_{i}$ is the frequency of the $i$ th allele, $f_{i j}$ the frequency of the genotype formed by the combination of the $i$ th and $j$ th alleles, and $W_{i j}$ the "fitness" (probability of survival) of that genotype, then

$$
W_{i}=\left(\Sigma_{j} f_{i j} W_{i \jmath}\right) / q_{i}
$$

is the mean fitness of the allele,

$$
\bar{W}=\Sigma_{i} q_{i} W_{i}=\Sigma_{i j} f_{i j} W_{i j}
$$

is the mean fitness of the population, and

$$
V_{H}=\Sigma_{i} q_{i}\left(W_{i}-\bar{W}\right)^{2}
$$

may be called the haploid variance in fitness. The $W_{i f}$ may be frequencydependent and $W_{i}$ and $\bar{W}$ may contain the inbreeding coefficient; some systems of assortative mating might also be included. 
The condition for equilibrium is that

$$
V_{H}=0
$$

That this gives equilibrium is easily seen when we remember that (Wright 1949)

$$
\Delta q_{i}=q_{i}\left(W_{i}-\bar{W}\right) / \bar{W}
$$

or that (4) implies $W_{i}=\bar{W}$ and hence

$$
W_{i}=W_{j}
$$

for all $i, j$. Under random mating $V_{H}$ is half the additive genetic variance determined from breeding values, and this special case of (4) is widely known.

A general adaptive topography with equilibria at its zero points can thus be drawn by plotting $V_{H}$ against allele frequencies. Like the $\bar{W}$ topography

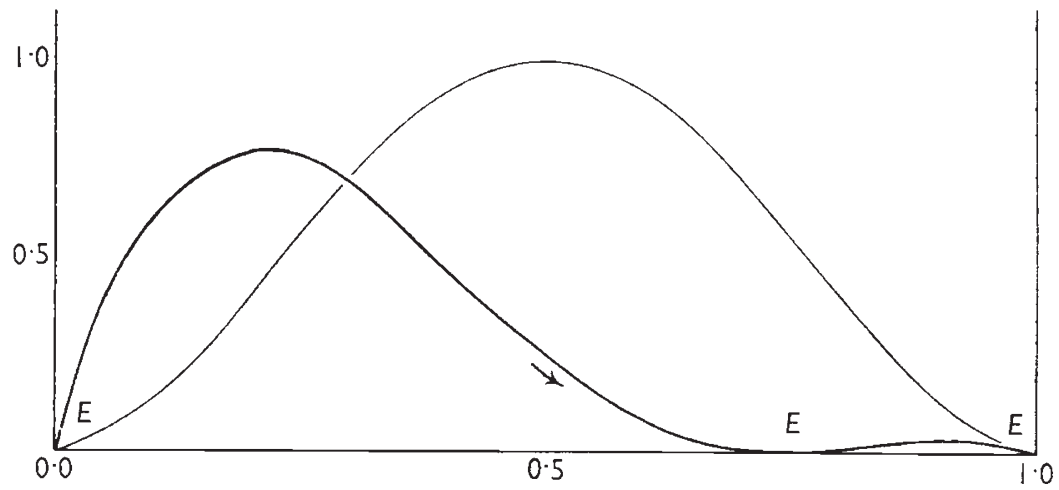

FIg. 1.-Haploid variance (thick line), dominance and variance (thin line) for two alleles, against gene frequency. Fitnesses are $W_{00}=1, W_{01}=4, W_{11}=3$. The equilibria $(E)$ are at the zero points of the haploid variance, and the central one is stable, the arrow indicating the direction of change in the population at gene frequency $\frac{1}{2}$.

it is most easily formed by an electronic computer. Like the $\bar{W}$ topography the graph is extremely difficult to draw when there are more than four alleles; with four alleles one can plot $V_{H}$ (or $\bar{W}$ ) inside a topologically distorted tetrahedron (Turner, 1967a). Fig. l shows the simplest case, two alleles with random mating.

In the $\bar{W}$ topography stable equilibria are at maxima, unstable at minima; the $V_{H}$ topography says nothing about stability, so that in addition to (4) we need the equation for the change in $V_{H}$. In general this is a polynomial, but the first (linear) term is sufficient for our needs. This is a special case of equation (Al7) in the appendix:

$$
\Delta V_{H}=\left\{M O(W)^{3}\right\} / \bar{W}+2 \Sigma_{i} q_{i}{ }^{2}\left(W_{i}-\bar{W}\right)^{2}\left(\partial W_{i} / \partial q_{i}-\partial \bar{W} / \partial q_{i}\right) / \bar{W}
$$

where

$$
\left\{M O(W)^{3}\right\}=\Sigma_{i} q_{i}\left(W_{i}-\bar{W}\right)^{3}
$$

is the third moment of fitness about the mean. The nature of the second term is most easily seen by reducing (7) to the equation for two alleles and 
constant selection when it becomes

$$
\Delta V_{H}=V_{H}\left\{\left(q_{1}-q_{0}\right)\left(W_{0}-W_{1}\right)-4 q_{0} q_{1} d_{w}\right\} / \bar{W}
$$

where $d_{w}$ is the dominance in fitness

$$
d_{w}=W_{01}-\frac{1}{2}\left(W_{00}+W_{11}\right)
$$

$q_{0}, q_{1}$ are the gene frequencies, and the meaning of the other subscripts is obvious. Equations similar to (7) and (9) are given by Kimura (1958) and Nei (1963).

Thus to determine the stability of the equilibria in the variance topography one need only determine the sign of (7) at a limited number of points in the topography; these will imply the direction of movement over the whole topography. For example, in fig. 1, the change of $V_{H}$ at gene frequencies $0.5,0.5$ has from (9) a sign opposite to that of $d_{w}$, so that if dominance is positive $V_{H}$ decreases and the non-trivial equilibrium $(E)$ is stable. (This, plus the fact that the graph of $V_{H}$ on $q$ gives a non-trivial minimum only if the fitness of the heterozygote lies outside the range of the homozygotes, gives the well-known condition that heterozygotes must be fittest.) For multiple alleles with inbreeding the term within the last bracket of $(7)$ is

$$
(1-F)\left\{\Sigma_{j} W_{i j}\left(\partial q_{j} / \partial q_{i}\right)-\Sigma_{i j} W_{i j}\left[q_{i}\left(\partial q_{j} / \partial q_{i}\right)+q_{j}\right]\right\}-F \Sigma_{j} W_{j j}\left(\partial q_{j} / \partial q_{i}\right)
$$

where $i \geqslant j, i j \neq j i$, and the derivatives are defined in the appendix. This will serve as an example of the more complex cases; frequency-dependence is similarly expressed in the derivatives.

\section{Special cases}

Any parameter $Y$, such that $V_{H}$ can be expressed as a function of a simple polynomial, with zero constant term, of $\partial Y / \partial q_{i}$ for all $i$, will maximise or minimise at equilibrium, and for a subset of such parameters this will be sufficient. Thus, for example, with two alleles under random mating and constant selection

$$
V_{H}=\frac{1}{4} q_{0} q_{1}\left(d \bar{W} / d q_{0}\right)^{2}
$$

whence $\bar{W}$ maximises or minimises. Therefore, an important aspect of the "fundamental theorem" can be derived from the theorem of decreasing variance. Likewise some of the various parameters which maximise under more complex systems turn out to be consequences of the variance theorem.

The simplest and, usually, quickest way of solving explicitly the nontrivial equilibria is also a special case. Equation (6) says that at equilibrium the rate of transmission of any one allele into the next generation must equal the rate of transmission of any other allele. Alternatively, we may say that the rate of removal of the allele, as a fraction of its total frequency, equals the same fraction for any other allele. Formally if $\delta q$ represents the rate of removal, then

$$
\left(\delta q_{i}\right) / q_{i}=\left(\delta q_{j}\right) / q_{j}
$$

For two alleles, with genotypes $A_{0} A_{0}, A_{0} A_{1}, A_{1} A_{1}$, with fitnesses $1-s_{0}, 1$, $1-s_{1}$ this gives immediately, for equilibrium

$$
\begin{gathered}
-s_{0} q_{\theta}{ }^{2} / q_{0}=-s_{1} q_{1}^{2} / q_{1} \\
\hat{q}_{0} / \hat{q}_{1}=s_{1} / s_{0}
\end{gathered}
$$


which is the well-known solution. Stability is tested by increasing the frequency of the allele on one side of (13); if that side becomes the larger the equilibrium is stable. Thus (14) becomes

$$
s_{0}\left(\hat{q}_{0}+\Delta q\right)^{2} /\left(\hat{q}_{0}+\Delta q\right): s_{1}\left(\hat{q}_{1}-\Delta q\right)^{2} /\left(\hat{q}_{1}-\Delta q\right)
$$

which reduces to

$$
s_{0} \Delta q:-s_{1} \Delta q \text {. }
$$

The LHS is greater and the equilibrium stable if both $s_{i}$ are positive.

Crow, who invented this method (Crow, 1958) did not extend it, but it does work very well for more elaborate cases. For example, three alleles, with selection coefficients against $A_{0} A_{0}, A_{0} A_{2}$ being $s_{00}, s_{02}$ and so on (no genotype with unit fitness), the simultaneous equations

$$
\begin{gathered}
\left(\delta q_{0}\right) / q_{0}=\left(\delta q_{1}\right) / q_{1}=\left(\delta q_{2}\right) / q_{2} \\
q_{0}+q_{1}+q_{2}=1
\end{gathered}
$$

solve by determinants to give

$$
\mathcal{J} \hat{q}_{0}=s_{11} s_{22}+s_{12}\left(s_{01}+s_{02}-s_{12}\right)-s_{11} s_{02}-s_{22} s_{01}
$$

and so symmetrically for the other alleles, where $\mathcal{F}$ is the sum of the RHS of the three equations. This is the same as the usual determinantal solution $(\mathrm{Li}, 1955 a)$, though not as elegant as that based on matrix algebra $(\mathrm{Li}$, 1967c). With inbreeding

$$
s_{0}\left\{(1-F) q_{0}^{2}+F q_{0}\right\} / q_{0}=s_{1}\left\{(1-F) q_{1}^{2}+F q_{1}\right\} / q_{1}
$$

yields

$$
\hat{q}_{0} / \hat{q}_{1}=\left(s_{1}-s_{0} F\right) /\left(s_{0}-s_{1} F\right) .
$$

Equilibrium between a mutation rate $\mu$ from $A_{0}$ to its recessive allele $A_{1}$ (fitness of $A_{1} A_{1}=1-s_{1}$ ) is approximately

$$
\begin{aligned}
-\mu q_{0} / q_{0} & =\left(\mu q_{0}-s_{1} q_{1}^{2}\right) / q_{1} \\
\hat{q}_{1}^{2} & =\mu / s_{1} .
\end{aligned}
$$

or

With gametic selection (heterozygotes segregating $(1-m) A_{0}$ gametes and $m A_{1}$ gametes) and random union of gametes, $(\delta q)$ is best treated as survival. Then

$$
\left\{\left(1-s_{0}\right) q_{0}^{2}+2 q_{0} q_{1}(1-m)\right\} / q_{0}=\left\{\left(1-s_{1}\right) q_{1}^{2}+2 q_{0} q_{1} m\right\} / q_{1}
$$

whence

$$
\hat{q}_{0} / \hat{q}_{1}=\left(s_{1}+1-2 m\right) /\left(s_{0}-1+2 m\right) .
$$

The above solutions, all known from other derivations, show that the $(\delta q)$ method is usually simpler and more direct than the use of $\Delta q$ equations or the use of " weights" ( $U$ method) (Lewontin, 1958).

\section{Complex cases}

If the population has two phases, with a common gene frequency, say males and females, then

$$
\left.\Delta q_{i}=\frac{1}{2} q_{i}\left\{W_{i M}-\bar{W}_{M}\right) / \bar{W}_{M}+\left(W_{i F}-\bar{W}_{F}\right) / \bar{W}_{F}\right\}
$$


and equilibrium is given by

$$
\frac{1}{2} \Sigma_{i} q_{i}\left\{\left(W_{i M}-\bar{W}_{M}\right) / \bar{W}_{M}+\left(W_{i F}-\bar{W}_{F}\right) / \bar{W}_{F}\right\}^{2}=0 .
$$

Similarly, when selection acts alternately on diploids and haploids and when zygosis is by random union of gametes (as in most plants) we have

$$
\Delta q_{i}=q_{i}\left(W_{i d}-\bar{W}_{d}\right) / \bar{W}_{d}+q_{i} W_{i d}\left(W_{i h}-\bar{W}_{h}\right) / \bar{W}_{d} \bar{W}_{h}
$$

and equilibrium when

$$
\Sigma_{i} q_{i}\left\{W_{i d}-W_{d}+W_{i d}\left(W_{i h}-\bar{W}_{h}\right) / \bar{W}_{h}\right\}^{2}=0,
$$

$d$ and $h$ indicating diploid and haploid.

Two loci with recombination is an example of a case in which the stable genotype distribution is not reached by one round of mating. There are two ways to solve this case: we may determine the equilibria which would occur if there was no recombination (by solving for four alleles at one locus) and examine qualitatively the way recombination moves the equilibria (Turner, $1967 a, b)$; or we may determine the equilibria exactly from the zero points of

$$
\Sigma_{i}\left\{q_{i}\left(W_{i}-\bar{W}\right)+k(i) R D W_{h}\right\}^{2}
$$

where $i=0,1,2,3$ are the gametes $a b, a B, A b, A B, R$ is the recombination fraction, $W_{h}$ is the fitness of double heterozygotes, the gametic excess is

and

$$
D=q_{0} q_{3}-q_{1} q_{2}
$$

$$
\begin{aligned}
& k(i)=-1 \text { if } i=0,3 \\
& k(i)=1 \text { if } i=1,2 .
\end{aligned}
$$

This method uses the variance in fitness of the smallest unit taking part in the mating system-not an allele nor a chromosome, but a length of chromosome partly broken by recombination.

No doubt a similar method could be devised for autopolyploids (see $\mathrm{Li}$, $1967 c)$.

Stability is probably best determined by finding the change in variance at selected gene frequencies by means of a simulated round of selection.

\section{Discussion}

Evolution by natural selection has been described as the " survival of the fittest"; this perhaps accounts for the popularity among population geneticists of maximisation theorems, seeming to be a mathematical expression of this idea (although the only author who has stated this explicitly is Blakley (1967)). But evolution by natural selection is not simply the survival of the fittest. It was well for evolutionists to emphasise the ability of selection to perfect the organism when they were battling with those who, following the vogue set by John Ray (1701), saw such perfection as evidence of Divine Love; this argument settled, we can see from such phenomena as hospitals and genetic loads that organisms are not perfect. (See Cain, 1964 for a most interesting review of the history of this idea.) Genetically speaking the fittest survive only until the end of the generation, when their genotypes are broken down in the mating system. Only an odd property of random mating tricks us into thinking the rule extends across the generations. The survival 
of the fittest is not a fundamental fact of evolution by natural selection and it is not surprising that it is difficult to formulate a fundamental theorem corresponding with this idea. The theorem proposed here, that variance minimises, is based on the simple idea that populations go on evolving until all the bits of genetic material which take part in the mating system are being handed on in equal proportions. I submit that it is more profitable to regard intra-population evolution in this almost too obvious way than to search for physically meaningful parameters which maximise.

This is not to say that if fitness is defined in a wider sense (utilisation of energy, ability to cope with a fluctuating environment), it will not be possible to formulate a maximisation law. Even within populations we might expect the breeding system to change in such a way as to maximise the more restricted "fitness"; that is, inbreeding should change to outbreeding, outbreeding to apomixis and loose linkage to tight linkage; but as, on the whole, this does not happen we must be cautious.

Nor need we denigrate the usefulness of the various maximising parameters for prediction in individual cases. Even the controversial case of two loci under random mating is very amenable to treatment by the maximisation of $\bar{W}$. In reply to Moran's criticisms of the $\bar{W}$ topography in this case (Moran, 1964), Wright has countered that the strictures make little difference to his shifting balance theory (Wright, 1965), and that most loci are unlinked and do not interact, so that as Kimura (1965) has shown, the $\bar{W}$ topography is an adequate approximation. But many loci are closely linked, and a fair proportion of these must interact in such a way as to invalidate the $\bar{W}$ topography; as $\mathrm{Li}(1967 c)$ says, an approximation valid for some cases does not excuse us from finding the general case. Kimura's (1966) demonstration that $\bar{W}$ is always maximal in a plane of constant crossproduct-ratio, only provides a general theorem if the population remains in that plane. But if the $\bar{W}$ topography is extended into another dimension, and an appropriate allowance made for the effects of recombination, we have a model which describes in general the equilibria in a two-locus polymorphism, adds a new factor to the shifting-balance theory, and predicts the evolution of close linkage (a theory which Fisher (1930) first propounded in the same book as his fundamental theorem, although both cannot in general be true simultaneously) (Turner, $1967 a, b)$. The extended $\bar{W}$ model also describes, by relating the variation of population path (with varying recombination) to the formation of the $\bar{W}$ hypersurface, the failure of a new allele to spread unless it is closely linked to another (Turner, unpublished). Such a model does not of course replace explicit equations (for the most recent study see Bodmer and Felsenstein, 1967) but merely gives us a framework for understanding them, and allows us to see whether or not the conclusions drawn from the equations are general (the equations can be solved only in special cases).

\section{Summary}

1. Evolution, if the mating system is taken into account, is not simply the "survival of the fittest" and the "fundamental theorem of natural selection" (that mean fitness increases) is therefore bound to run into difficulties.

2. A basic theorem is that the haploid variance in fitness decreases to zero under selection (the decrease is not monotonic). Most selection 
theorems are special cases of this theorem, as is a very simple method for finding the non-trivial equilibria.

3. An " adaptive topography" based on the theorem will predict the course of evolution even in quite complex cases, and is not restricted, as is the mean fitness topography, to the simplest conditions.

4. Equations are given for the change of mean and variance of metric characters (including fitness as a special case) under selection.

Acknowledgments.-I am grateful to Professor M. H. Williamson and Professor A. Robertson, F.R.S. for their helpful critique of a draft of this paper, and to Professor M. S. Bartlett, F.R.S. for a discussion of Taylor's expansion.

\section{ApPENDix: GHANGES OF MEAN AND VARIANGE OF METRIC GHARACTERS UNDER SELEGTION}

Here is derived, in a general form, the equation for the change of variance (7) and also several other equations of general interest which use the same proof. First note the definitions of the partial derivatives of allele frequencies (Wright, 1955)

$$
\partial q_{i} / \partial q_{i}=1, \partial q_{j} / \partial q_{i}=-q_{j} /\left(1-q_{i}\right)
$$

The change in any population parameter, $Z$, with changing gene frequency can be calculated from the infinite expansion

$\Delta Z=\Sigma_{i}\left(\Delta q_{i}\right) \frac{\partial Z}{\partial q_{i}}+\Sigma_{i j} \frac{\left(\Delta q_{i}\right)\left(\Delta q_{j}\right)}{2 !} \frac{\partial^{2} Z}{\partial q_{i} q_{j}}+\Sigma_{i j k} \frac{\left(\Delta q_{i}\right)\left(\Delta q_{j}\right)\left(\Delta q_{k}\right)}{3 !} \frac{\partial^{3} Z}{\partial q_{i} q_{j} q_{k}} \ldots$

\section{$(i \geqq j \geqq k)$}

Take a metric character, values $X_{i j}$ in diploids, so that the mean for an allele is

$$
X_{i}=\Sigma_{j} f_{i j} X_{i j} / q_{i}
$$

and the population mean and haploid variance are given by

$$
\begin{gathered}
\bar{X}=\Sigma_{i j} f_{i j} X_{i j}=\Sigma_{i} q_{i} X_{i} \\
V_{H(X)}=\Sigma_{i} q_{i}\left(X_{i}-\bar{X}\right)^{2}
\end{gathered}
$$

having a haploid covariance with fitness

$$
\left\{\operatorname{covar}_{H}(W, X)\right\}=\Sigma_{i} q_{i}\left(W_{i}-\bar{W}\right)\left(X_{i}-\bar{X}\right)
$$

We first establish the rule for taking the derivatives in (A2) by determining the change in mean for two alleles under random mating and with no dominance. In this case we need use only one gene frequency in the derivative (as the other is automatically determined), so that

$$
\Delta q_{0}=\left(q_{0} q_{1} / 2 \bar{W}\right)\left(d \bar{W} / d q_{0}\right)
$$

and the haploid covariance with fitness is

$$
\left\{\operatorname{covar}_{H}(W, X)\right\}=\frac{1}{4} q_{0} q_{1}\left(d \bar{W} / d q_{0}\right)\left(d \bar{X} / d q_{0}\right) .
$$

Substitution of $\bar{X}$ for $Z$ in (A2) yields at once

$$
\Delta \bar{X}=2\left\{\operatorname{covar}_{H}(W, X)\right\} / \bar{W}
$$


To obtain this result by summing

$$
\Delta \bar{X}=\left(\Delta q_{0}\right)\left(\partial \bar{X} / \partial q_{0}\right)+\left(\Delta q_{1}\right)\left(\partial \bar{X} / \partial q_{1}\right)
$$

using rule (Al) and equation (5) it is necessary to differentiate only that part of $\bar{X}$ which is a direct function of $q_{i}$. That is, (A10) must be written

$$
\Delta \bar{X}=\left(\Delta q_{0}\right)\left\{\partial\left(q_{0} X_{0}\right) / \partial q_{0}\right\}+\left(\Delta q_{1}\right)\left\{\partial\left(q_{1} X_{1}\right) / \partial q_{1}\right\}
$$

By symmetry and induction this applies to any number of alleles. Also by symmetry and induction (for details of the method, see Turner, 1967b, appendix III) the change in $X$ for any number of alleles without dominance will be twice the haploid covariance with fitness (divided by $\bar{W}$ ), and the first term of the expansion is therefore

$$
\Delta \bar{X}=\Sigma_{i}\left(\Delta q_{i}\right)\left\{\partial\left(q_{i} X_{i}\right) / \partial q_{i}\right\}=2\left\{\operatorname{covar}_{H}(W, X)\right\} / \bar{W}
$$

for any number of alleles.

The same rule of differentiation applies to the variance, and to the second derivatives.

Under random mating, but including dominance, we introduce the second term of (A2), all higher terms being zero. Thus we have finally

$\Delta \bar{X}=2\left\{\operatorname{covar}_{H}(W, X)\right\} / \bar{W}$

$$
+\left\{\Sigma_{i} q_{i}^{2}\left(W_{i}-\bar{W}\right)^{2} X_{i i}+2 \Sigma_{i j} q_{i} q_{j}\left(W_{i}-\bar{W}\right)\left(W_{j}-\bar{W}\right) X_{i j}\right\} / \bar{W}^{2} \quad(i i \neq i j)
$$

which for two alleles reduces to

$$
\Delta \bar{X}=2\left[\left\{\operatorname{covar}_{H}(W, X)\right\}-2 V_{H} q_{0} q_{1} d_{X} / \bar{W}\right] / \bar{W}
$$

where

$$
d_{X}=X_{01}-\frac{1}{2}\left(X_{00}+X_{11}\right)
$$

is the dominance of the character and $V_{H}$ is the haploid variance in fitness. (A14) may also be obtained directly by the method of (A7-10).

Thus the change in the mean of a metric character equals its haploid covariance with fitness (divided by $\bar{W}$ ) (this is not the directly measurable diploid covariance) minus a correction for dominance ( $c f$. Falconer, 1966). The change in mean fitness itself is readily shown to be (from A14) for two alleles

$$
\Delta \bar{W}=V_{H} / \bar{W} \times\left(\bar{W}+\bar{W}_{I}\right) / \bar{W}
$$

where $\bar{W}_{I}=\Sigma_{i} q_{i} W_{i i}$. This is not in general true for three or more alleles (C. C. Li, personal communication) contrary to my former opinion (Turner, $1967 b)$; the exact equation for $\Delta \bar{W}$ with any number of alleles is clearly (A13) with $X$ replaced by $W$.

The change in the haploid variance of the character is approximately, using the same rule of differentiation as for the mean, and taking only the first term of (A2):

$$
\begin{aligned}
\Delta V_{H(X)} \simeq & \Sigma_{i}\left(\Delta q_{i}\right)\left\{\partial q_{i}\left(X_{i}-\not{X}\right)^{2} / \partial q_{i}\right\} \\
= & \Sigma_{i}\left[\left\{q_{i}\left(W_{i}-\bar{W}\right) / \bar{W}\right\} \times\left\{\left(\partial q_{i} / \partial q_{i}\right)\left(X_{i}-\bar{X}\right)^{2}\right.\right. \\
= & \left.\left.\quad+2 q_{i}\left(X_{i}-\bar{X}\right)\left[\partial\left(X_{i}-\not{X}\right) / \partial q_{i}\right]\right\}\right] \\
= & \left.\operatorname{como}_{H}\left(W, X^{2}\right)\right\} / \bar{W} \\
& +2 \Sigma_{i} q_{i}{ }^{2}\left(W_{i}-\bar{W}\right)\left(X_{i}-\bar{X}\right)\left(\partial X_{i} / \partial q_{i}-\partial X / \partial q_{i}\right) / \bar{W}
\end{aligned}
$$


or for two alleles

$$
\Delta V_{H(X)} \simeq\left\{\operatorname{covar}_{H}(W, X)\right\}\left\{\left(q_{1}-q_{0}\right)\left(X_{0}-X_{1}\right)-4 q_{0} q_{1} d_{X}\right\} / \bar{W}
$$

where

$$
\left\{\operatorname{como}_{H}\left(W, X^{2}\right)\right\}=\Sigma_{i} q_{i}\left(W_{i}-\bar{W}\right)\left(X_{i}-\bar{X}\right)^{2} .
$$

The derivatives in (A17) follow rule (Al), and involve the whole of $\bar{X}$, not merely $q_{i} X_{i}$. (A17-18) therefore state that the change in the haploid variance of a character is approximately equal to a comoment with fitness, minus a correction for dominance; this approximation is true for any conditions of selection and mating. These equations reduce to (7) and (9) when the character considered is fitness itself.

The exact equations for the change in variance are in general very elaborate, but that for the haploid variance in fitness at two alleles under constant selection and random mating involves only the first four terms of (A2) and is

$$
\begin{aligned}
& \Delta V_{H}=\frac{V_{H}}{\bar{W}}\left\{\left(q_{1}-q_{0}\right)\left(W_{0}-W_{1}\right)-4 q_{0} q_{1} d_{w}\right. \\
&+\frac{V_{D}-V_{H}-4\left(q_{1}-q_{0}\right)\left(W_{0}-W_{1}\right) q_{0} q_{1} d_{w}}{\bar{W}} \\
&+\frac{\left(q_{1}-q_{0}\right)\left(W_{0}-W_{1}\right) V_{D}+4 V_{H} q_{0} q_{1} d_{w} / 3}{\bar{W}^{2}} \\
&\left.-\frac{V_{H} d_{w}}{\bar{W}^{3}}\right\}
\end{aligned}
$$

where $V_{D}$ is the dominance variance in fitness, and $d_{w}$ is defined in (10). The first term of this is of course equation (9). Similarly for the dominance variance:

$$
\begin{aligned}
\Delta V_{D}=\frac{V_{D}}{\bar{W}}\left\{2\left(q_{1}-q_{0}\right)\left(W_{0}-W_{1}\right)+\frac{\left(q_{0}^{2}+q_{1}^{2}\right)\left(W_{0}-W_{1}\right)^{2}}{\bar{W}}+\frac{2}{3} \frac{M O(W)^{3}}{W^{2}}+\right. \\
\left.\frac{1}{3} \frac{V_{D} V_{H^{2}}}{\bar{W}^{3}}\right\}
\end{aligned}
$$

(See (8) for $M O(W)^{3}$.) Nei (1963) gives the first terms of (A20-21). The equation given by O'Donald (1967) for the change of variance in the absence of dominance (namely $\Delta V_{H}=-V_{H}^{2} / \bar{W}^{2}$ ) is equal to the second (quadratic) term of (A20). Warburton (1967) assuming no dominance and $\bar{W}=1$, found the first two terms of (A20). All these authors use the additive variance, so that (A20) is actually half the value which they obtain.

\section{REFERENGES}

BLAKLEY, G. R. 1967. Darwinian natural selection acting within populations. $\mathcal{F}$. theoret. Biol., 17, 252-281.

BODMER, W. F., AND FELSENSTEIN, J. 1967. Linkage and selection: theoretical analysis of the deterministic two locus random mating model. Genetics, 57, 237-265.

CAIN, A.J. 1964. The perfection of animals. Viewpoints in Biology, 3, 36-63, (ed. Carthy, J. D. and Dunnington, C. L.). London, Butterworths.

CROW, J. F. 1958. Some possibilities for measuring selection intensities in man. Amer. Anthropol., 60, 1-13. (Memoir No. 86) 
FALCONER, D. S. 1961. Introduction to quantitative genetics. Edinburgh and London, Oliver and Boyd.

FALCONER, D. s. 1966. Genetic consequence of selection pressure. In Genetic and environmental factors in human ability, pp. 219-232, (eds. J. E. Meade and A. S. Parkes). Edinburgh and London, Oliver and Boyd.

FISHER, R. A. 1930. The genetical theory of natural selection. Oxford, Clarendon Press.

KIMURA, м. 1958. On the change of population fitness by natural selection. Heredity, 12, 145-167.

Kimura, M. 1966. Two loci polymorphism as a stationary point. Ann. Rep. Nat. Inst. Genet. Japan, 17, 65-67.

LEWONTTN, R. C. 1958. A general method for investigating the equilibrium of gene frequency in a population. Genetics, 43, 419-434.

LEWONTIN, R. C., AND WHITE, M. J. D. 1960. Interaction between inversion polymorphisms of two chromosome pairs in the grasshopper, Moraba scurra. Evolution, 14, 116-129.

LI, c. c. 1955a. The stability of an equilibrium and the average fitness of a population. Amer. Nat., 89, 281-295.

LI, c. c. 1955b. Population genetics. Chicago and London, Chicago University Press.

LI, c. c. 1967a. Fundamental theorem of natural selection. Nature, London, 214, 505-506.

LI, c. c. 1967b. The maximisation of average fitness by natural selection for a sex-linked locus. Proc. Nat. Acad. Sci., Washington, 57, 1260-1261.

LI, c. c. 1967c. Genetic equilibrium under natural selection. Biometrics, 23, 397-484.

MORAN, P. A. P. 1964. On the non-existence of adaptive topographies. Ann. hum. Genet., London, 27, 383-393.

NEI, M. 1963. Effects of selection on the components of genetic variance. Statistical Genetics and Plant Breeding (Nat. Acad. Sci.-Nat. Res. Counc. publ. 982, Washington), 501-515.

o'DONALD, P. 1967. On the evolution of dominance, over-dominance and balanced polymorphism. Proc. Roy. Soc., London, B268, 216-228.

RAY, J. 1701. The Wisdom of God manifested in the Works of the Creation, in two parts. Third edition. London, S. Smith and B. Walford.

TURNER, J. R. G. 1967a. On supergenes. I. The evolution of supergenes. Amer. Nat., 101, 195-221.

TURNER, J. R. G. 1967b. Mean fitness and the equilibria in multilocus polymorphisms. Proc. Roy. Soc., London, B169, 31-58.

WARBURTON, F. E. 1967. Increase in the variance of fitness due to selection. Evolution, 21, 197-198.

WRIGHT, s. 1949. Adaptation and selection. Genetics, palaeontology and evolution, pp. 365-389, (eds. Jepsen, G. L., Mayr, E. and Simpson, G. G.), Princeton Univ. Press.

wRIGHT, s. 1955. Classification of the factors of evolution. Cold Spring Harbor Symp. Quant. Biol., 20, 16-24D.

WRIGHT, s. 1965. Factor interaction and linkage in evolution. Proc. Roy. Soc., London, B162, 80-104. 